\title{
The Werner syndrome protein has separable recombination and survival functions ${ }^{\text {is }}$
}

\author{
Cristina Swanson $^{\mathrm{a}, 1}$, Yannick Saintigny ${ }^{\mathrm{a}, 1,2}$, Mary J. Emond ${ }^{\mathrm{b}}$, Raymond J. Monnat, Jr. ${ }^{\mathrm{a}, \mathrm{c}, *}$ \\ a Department of Pathology, University of Washington, Box 357705, Seattle, WA 98195-7705, USA \\ ${ }^{\mathrm{b}}$ Department of Biostatistics, University of Washington, Seattle, WA, USA \\ ${ }^{\mathrm{c}}$ Department of Genome Sciences, University of Washington, Seattle, WA, USA
}

Accepted 20 January 2004

\begin{abstract}
The Werner syndrome (WS) protein WRN is unique in possessing a $3^{\prime}$ to $5^{\prime}$ exonuclease activity in addition to the $3^{\prime}$ to $5^{\prime}$ helicase activity characteristic of other RecQ proteins. In order to determine in vivo functions of the WRN catalytic activities and their roles in Werner syndrome pathogenesis, we quantified cell survival and homologous recombination after DNA damage in cells expressing WRN missense-mutant proteins that lacked exonuclease and/or helicase activity. Both WRN biochemical activities were required to generate viable recombinant daughter cells. In contrast, either activity was sufficient to promote cell survival after DNA damage in the absence of recombination. These results indicate that WRN has recombination and survival functions that can be separated by missense mutations. Two implications are that Werner syndrome most likely results from the loss of both activities and their associated functions from patient cells, and that $W R N$ missense mutations or polymorphisms could promote genetic instability and cancer in the general population by selectively interfering with recombination in somatic cells.
\end{abstract}

(C) 2004 Elsevier B.V. All rights reserved.

Keywords: Werner syndrome; RecQ; Genetic instability; Homologous recombination

\section{Introduction}

Werner syndrome (WS) is a human autosomal recessive genetic instability syndrome. The intense interest in this uncommon disease has focused on the appearance of premature aging in affected individuals, together with an elevated risk of important age-associated diseases such as cancer, atherosclerotic cardiovascular disease, diabetes mellitus and osteoporosis [1,2]. Cancer and cardiovascular disease are the leading causes of premature morbidity and death in WS patients, in whom the mean age of death is $\sim 47$ years [3,4].

\footnotetext{
is Supplementary data associated with this article can be found, in the online version, at doi:10.1016/j.dnarep.2004.01.002.

* Corresponding author. Tel.: +1-206-616-7392; fax: +1-206-543-3967.

E-mail addresses: swansonc@u.washington.edu (C. Swanson), yannick.saintigny@cea.fr (Y. Saintigny), emond@u.washington.edu (M.J. Emond), monnat@u.washington.edu (R.J. Monnat Jr.).

1 These authors contributed equally to this work.

2 Present address: LMR-UMR CEA/CNRS 217, 92265 Fontenay aux Roses, Cedex, France.
}

Werner syndrome patients have mutations in the chromosome 8p12 WRN locus that encodes a 1432 residue, $162 \mathrm{kDa}$ member of the human RecQ helicase family [5]. RecQ helicase proteins are widely distributed in nature, and there is good evidence that RecQ helicases play important roles in genome stability maintenance in prokaryotes, in single cell eukaryotes and in mammals [6,7]. The role of human RecQ helicases in genome stability assurance and tumor suppression was highlighted by the identification of Werner syndrome, Bloom syndrome and Rothmund-Thomson syndrome: each of these genetic instability/tumor predisposition syndromes results from loss of function of a different human RecQ helicase protein [7,8].

Biochemical characterization of the human WRN RecQ helicase protein has revealed a unique $3^{\prime}$ to $5^{\prime}$ exonuclease activity in addition to the $3^{\prime}$ to $5^{\prime}$ helicase and ATPase activities characteristic of other RecQ helicase proteins $[9,10]$. Among the WRN mutations that have been identified in WS patients, none selectively inactivates the WRN exonuclease or helicase activities [11]. Thus, the functional importance of these biochemical activities is uncertain, and it is not 


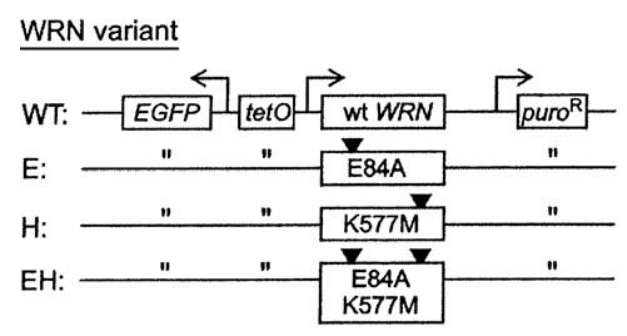

(A)

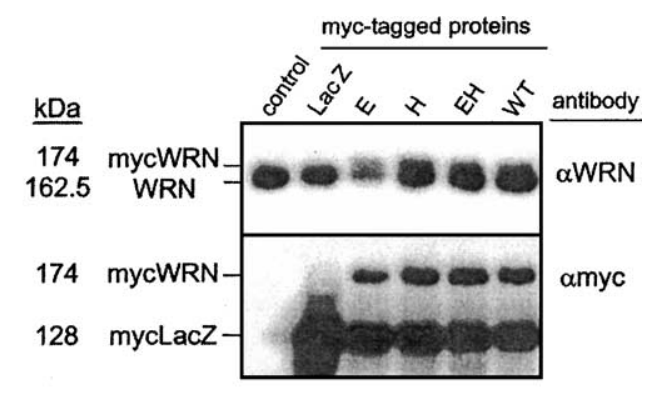

(B)

Fig. 1. Transgene expression and detection of genetically characterized WRN proteins. (A) Vectors encoding N-terminal myc epitope-tagged wildtype (WT), exonuclease-deficient $(\mathrm{E})$, helicase-deficient $(\mathrm{H})$ or double missense $(\mathrm{EH}) \mathrm{WRN}$. Altered WRN residues are numbered and changes are indicated in single letter amino acid code. Key: $E G F P$, enhanced green fluorescent protein gene; tet $O$, tetracycline operator sequence; puro ${ }^{R}$, puromycin resistance gene. (B) Control GM639 fibroblast cells were transiently transfected with WRN plasmids in A together with a tTA transactivator plasmid [14]. Transgene-encoded WRN proteins $(174 \mathrm{kDa})$ were detected with WRN (upper panel) or myc epitope (lower panel) antibodies. A myc epitope-tagged $128 \mathrm{kDa}$ bacterial $\beta$-galactosidase encoded by pCS2 + cßlacZ plasmid [40] was used as a positive control for Westerns and for transfection efficiency. Protein extracts were prepared $24 \mathrm{~h}$ after transfection.

known whether both must be lost to promote WS disease pathogenesis.

We recently identified a role for the human WRN protein in cell survival and homologous recombination after DNA damage $[12,13]$. In order to determine whether one or both of the WRN catalytic activities were required for in vivo function, we quantified cell survival and recombination after DNA damage using cells that expressed wildtype WRN or missense-mutant forms of WRN that lacked exonuclease, helicase or both enzymatic activities (E84A, K577M, and E84A/K577M WRN, respectively; Fig. 1). We found that recombination required both of the WRN biochemical activities. Either activity alone, in contrast, was able to promote cell survival after DNA damage in the absence of recombination. Our results provide new information on WRN function in vivo and on the pathogenesis of Werner syndrome, and suggest new ways in which $W R N$ mutation or variation may be promoting disease risk in the general population.

\section{Materials and methods}

\subsection{Cell lines and cell culture}

Werner syndrome and control SV40 fibroblast cell lines used for these analyses were from unrelated individuals and have been previously described $[12,13]$. Both WS cell lines contain WRN mutations and do not make detectable WRN protein. Cells were grown in Dulbecco-modified Eagle's Medium (DMEM, $4.5 \mathrm{gm} / 1$ glucose; Cellgro) supplemented with $10 \%$ (v/v) fetal bovine serum (FBS; Hyclone), $100 \mathrm{U} / \mathrm{ml}$ penicillin $\mathrm{G}$ sulfate, and $100 \mu \mathrm{g} / \mathrm{ml}$ streptomycin sulfate in a humidified $37^{\circ} \mathrm{C}, 7 \% \mathrm{CO}_{2}$ incubator.

\subsection{WRN expression vectors}

Plasmids expressing divergently transcribed cDNAs encoding myc epitope-tagged WRN proteins or enhanced green fluorescent protein were constructed from pBI (Clontech; Fig. 1). Transient transfections were performed using SuperFect (Qiagen) according to the manufacturer's protocol: cells $\left(1-5 \times 10^{5} / 10 \mathrm{~cm}\right.$ dish $)$ were plated $48 \mathrm{~h}$ prior to co-transfection with $10 \mu \mathrm{g}$ of WRN expression vector $\pm 4 \mu \mathrm{g}$ of a tet-transactivator (tTA) coding plasmid pUHD15-1 [14]. Stable transfections also were performed using SuperFect: $2-8 \times 10^{5}$ cells $/ 6 \mathrm{~cm}$ dish were transfected with $5 \mu \mathrm{g}$ of expression vector, then dilute-plated $24 \mathrm{~h}$ after transfection in $10 \mathrm{~cm}$ dishes in order to recover puromycin-resistant $(0.1-0.4 \mu \mathrm{g} / \mathrm{ml})$ colonies.

\subsection{Western blot detection of WRN}

Cell pellets $\left(3 \times 10^{7}\right.$ cells $\left./ \mathrm{ml}\right)$ were resuspended in lysis buffer $(25 \mathrm{mM}$ Tris pH $8,5 \mathrm{mM}$ EDTA, $600 \mathrm{mM} \mathrm{NaCl}$, $10 \%$ glycerol, $0.01 \mathrm{mM}$ DTT, $0.1 \%$ NP-40), incubated on ice for $40-60 \mathrm{~min}$, then centrifuged to remove cell debris. Proteins were separated by SDS-PAGE electrophoresis, and then electroblotted onto PVDF membrane $(100 \mathrm{~V}, 90 \mathrm{~min})$ in $25 \mathrm{mM}$ Tris, $192 \mathrm{mM}$ glycine buffer containing $20 \%$ (v/v) methanol. Non-specific antibody binding was blocked by incubation in TBS-T buffer $(150 \mathrm{mM} \mathrm{NaCl}, 10 \mathrm{mM}$ Tris $\mathrm{pH}$ $8,0.05 \%$ Tween-20) containing $10 \%$ (w/v) non-fat dry milk (NFDM). WRN was detected with a mouse monoclonal $\mathrm{IgG}_{1}$ anti-WRN antibody (BD, Transduction Laboratories; 1:1000 dilution). Myc epitope-tagged proteins were detected with 9E10 anti-myc epitope tag-specific $\mathrm{IgG}_{1}$ hybridoma supernatant [15] at a 1:50 dilution. Bound antibodies were detected with a goat anti-mouse $\mathrm{IgG}_{1}$-HRP antibody conjugated to horseradish peroxidase (Southern Biotechnology Associates, Inc.; 1:2000 dilution) and chemiluminescence detection (ECL + plus, Amersham Biosciences).

\subsection{Cell survival and recombination assays}

Survival and recombination assays were performed as previously described [13]. Cells were transfected $48 \mathrm{~h}$ 
prior to DNA damage in order to allow WRN protein expression; treated with cis-Pt; and then replated $24 \mathrm{~h}$ later in order to determine colony forming efficiency (CFE; $100-1000$ cells $/ 6 \mathrm{~cm}$ well) or recombinant colony formation $\left(10^{5}-10^{6}\right.$ cells $/ 10 \mathrm{~cm}$ dish). CFE plates were grown for 10 days, and recombinant colonies for 10-16 days in the presence of $400-600 \mu \mathrm{g} / \mathrm{ml} \mathrm{G} 418$ (Gibco/BRL), prior to staining and counting. Transient transfections were normalized for the percent transfected cells as revealed by use of a co-transfected bacterial $\beta$-galactosidase reporter plasmid. Recombination frequencies were corrected for the intrinsic difference in CFE between WRN-deficient and control cells, and for the selective sensitivity of WRN cells to $c i s$-Pt cytotoxicity $([13,21]$ and additional unpublished results). The statistical significance of differences in survival or recombinant colony generation after DNA damage as a function of genotype and WRN protein expression was determined as previously described [13].

\section{Results}

In order to determine the requirement for WRN exonuclease and helicase activity in vivo, we expressed wildtype or missense-mutant WRN protein in WS or control cells, and then quantified cell survival and recombination after DNA damage. Four different WRN proteins were expressed: wildtype WRN possessing exonuclease and helicase activity, and WRN missense-mutants that lacked exonuclease or helicase activity (E84A or K577M WRN, respectively) or both activities (E84A/K577M WRN; Fig. 1A). The missense mutations used had been previously shown to inactivate the WRN helicase or exonuclease activity [16,17]. A myc epitope tag was added in-frame to the N-terminus of all of the WRN proteins to allow unambiguous detection of transgene-encoded proteins in the presence of native WRN (Fig. 1B). This epitope tag does not interfere with either biochemical activity of WRN or with in vivo functioning of WRN $[13,18]$.

The cells used for experiments were SV40-transformed fibroblasts from WS patients or controls. We had previously demonstrated a mutator phenotype and recombination deficits in these cells, as well as selective drug sensitivities that parallel the phenotype of primary WS cells $[13,18]$. One potentially attractive alternate cell type for the assays described here are telomerase-immortalized WS fibroblasts [19]. Although these cells proliferate indefinitely, we chose not to use them as they no longer faithfully recapitulate the mutator phenotype or selective drug sensitivity profiles observed in primary WS fibroblasts, peripheral blood lymphocytes or B-lymphoblastoid cell lines ([20]; additional results not shown).

The DNA damaging agent chosen for our analyses was cis-Pt, one of several DNA cross-linking agents to which WS patient cells and cell lines are selectively hypersensitive [21]. In contrast to several other cross-linking agents, cis-Pt does not require metabolic activation, and has a well-defined
DNA damage and mutational spectrum profile (see, for example, [22]). The dose and exposure time used in the experiments reported here $(2 \mu \mathrm{M}$ cis-Pt for $24 \mathrm{~h})$ was chosen on the basis of an extensive series of dose-response and exposure time experiments that revealed consistent, highly significant reductions in cell survival as measured by colony forming efficiency and by colony size distribution (CSD) assays as well as markedly reduced recombination in the absence of WRN function. The survival difference after $c i s$-Pt damage was marked: $\mathrm{LD}_{10}$ values for WRN and control cells were $1.0 \mu \mathrm{M}$ versus $2.1 \mu \mathrm{M}$, respectively, in experiments that examined dose-dependent killing over a three-log range ([13] additional data not shown).

The survival of control cells after cis-Pt damage, as measured by colony-forming efficiency, was unaffected by the transient expression of wildtype, single- or doublemissense-mutant forms of WRN $(P=0.43-0.87$; Fig. 2A, open bars). In contrast, WS cells had colony forming efficiencies that were significantly lower than control cells prior to and after DNA damage (Fig. 2A, compare C columns; $P=1.4 \times 10^{-6}$ ). The expression of wildtype WRN or of either single missense-mutant WRN protein

(A)
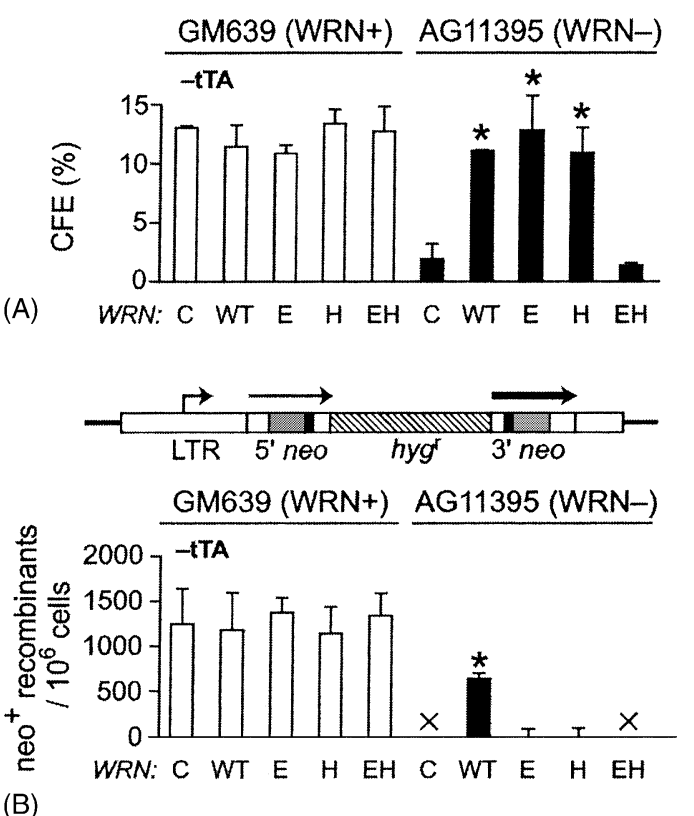

Fig. 2. WRN exonuclease or helicase activities can promote cell survival in the absence of recombination. (A) Survival of control and WS cells measured by colony forming efficiency after WRN expression and cis-Pt damage. The improved survival of WS cells expressing WT, E or $\mathrm{H}$ WRN was statistically significant (*, see text for $P$ values). (B) Upper panel: structure of the $\mathrm{pNeoA}$ recombination reporter plasmid. Arrows indicate direct repeat neomycin phosphotransferase (neo) genes inactivated by linker insertions (filled boxes), with crosshatch indicating the region of homology between linker insertion sites. key: LTR, retroviral long terminal repeat promoter; $h y g^{r}$, hygromycin resistance cassette. Bottom panel: frequency of neo ${ }^{+}$recombinant colonies $/ 10^{6}$ viable cells after cis-Pt damage. Only WT WRN protein led to a significant (*) recovery of neo+ recombinants when expressed in WS cells. Error bars are standard deviations for a minimum of two replicate experiments. 
in WS cells led to a significant increase in cell survival after DNA damage (Fig. 2A, right panel, filled bars; compare WT, E or $\mathrm{H}$ with $\mathrm{C} ; P=3.2-7.2 \times 10^{-6}$ ). These survival levels were indistinguishable from comparably transfected control cells (Fig. 2A, open bars; $P=0.30-0.98)$. In contrast, the expression of double missense-mutant WRN did not detectably improve the survival of WS cells (Fig. 2A, right panel, compare $\mathrm{C}$ and $\mathrm{EH}$; $P=0.96$ ).

Recombination in transiently transfected WS and control cells was measured by determining the frequency of recombination-dependent neo $^{+}$allele generation from a chromosomally integrated recombination reporter plasmid (pNeoA; Fig. 2B, top panel; [13,23]). Control cells display a $\sim 100$-fold increase in the frequency of neo ${ }^{+} / \mathrm{G} 418$-resistant recombinant colonies per surviving cell after DNA damage as compared with WS cells (Fig. 2B, bottom panel left; [13] and additional results not shown). No difference in recombinant frequency was observed in control cells transfected with a related control plasmid, or expressing any of the four WRN proteins shown in Fig. $1(P=0.73-0.88)$. In WS cells a statistically significant increase in recombinant colony formation after cis-Pt damage was observed only in cells that expressed wildtype WRN protein (Fig. 2B, bottom panel right, compare $\mathrm{C}$ and WT; $P=0.03$; see also Fig. 1 in Supplementary Material).

Cell survival and recombination after DNA damage were also analyzed in clonal derivatives of control or WS cells that stably expressed wildtype or missense-mutant WRN protein (Fig. 3). Control cells that stably expressed singleor double-missense-mutant forms of WRN had survival frequencies that were indistinguishable from cells transfected with a control plasmid or that expressed wildtype WRN (Fig. 3A, left panel open bars; additional results not shown). In contrast, WS cells that stably expressed either single missense-mutant form of WRN had significantly improved cell survival frequencies after $c i s$-Pt damage (Fig. 3A, right panel filled bars $\mathrm{E}$ or $\mathrm{H}$ versus EH; $P=9 \times 10^{-13}$ ) to levels that were indistinguishable from control cells (compare E or H CFEs of control cells with WRN-complemented WS cells; $P=0.19$ and 0.53 , respectively). WS cells that stably expressed double missense-mutant WRN had the same survival as untransfected WS cells lacking WRN protein $(P=$ $0.78)$. None of the stably expressed missense-mutant WRN proteins promoted the generation of neo ${ }^{+} / \mathrm{G} 418$-resistant recombinant colony formation after DNA damage (Fig. 3B; additional results not shown).

Western blot analyses indicated a range of WRN transgene expression levels in cells used for functional analyses (Figs. 4 and 5). In order to determine whether level of expression of WRN proteins also influenced cell phenotype after DNA damage, we quantified cell survival and recombination in control and WS cells after inducing WRN expression with tet-transactivator protein [14]. These experiments took advantage of the expression vector construction strategy that placed the WRN open reading frame un-
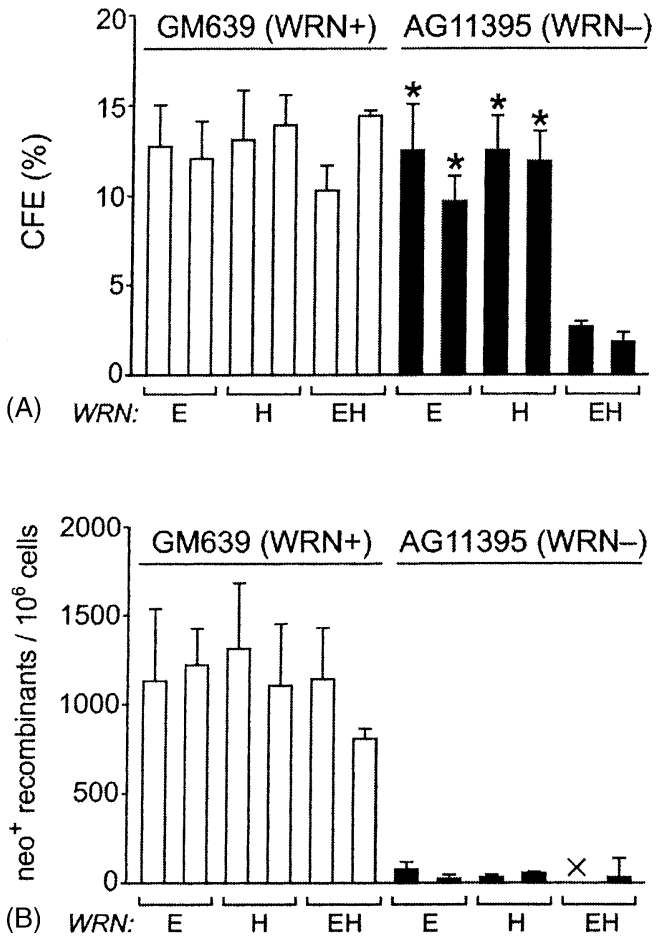

Fig. 3. Stable expression of WRN lacking exonuclease or helicase activity promotes cell survival but not recombination. (A) The colony forming efficiency of independent, WS cell clones expressing $\mathrm{E}$ and $\mathrm{H}$ WRN was significantly higher (*, see text for $P$ values) than the survival of WS-deficient cells expressing EH (catalytically inactive) WRN. (B) Frequency of neo ${ }^{+}$recombinant colonies $/ 10^{6}$ viable cells after cis-Pt damage. None of the WRN mutant proteins led to detectable recovery of neo $^{+}$recombinant colonies (see also Fig. 1 in Supplementary Material).

der the control of a CMV minimal promoter and adjacent to a tet repressor binding site (Fig. 1A). The rationale for these experiments was provided by previous work showing intermediate sensitivity of WRN heterozygous lymphoblastoid cell lines to several DNA damaging agents including cis-Pt $[24,25]$ and the identification of genetic instability in vivo in the red blood cell lineage of WRN heterozygotes [26].

Although $W R N$ transgene expression could be induced by tTA expression in both transient and stable transfection experiments to $50 \%$ of wildtype levels (Fig. 5A; additional results not shown), we observed no difference in cell survival or in recombination as a function of higher expression of any WRN protein (Fig. 5B; compare with Fig. 2A and B). These results indicate that WRN can promote cell survival even when expressed at low levels, whereas even high level expression of WRN lacking helicase or exonuclease activity cannot promote recombination in the absence of missing WRN catalytic activities. The apparent copy number independence of WRN function in our experiments may reflect the comparatively high level of WRN protein in fibroblast cell lines [27] and/or attenuated DNA damage checkpoint functioning in SV40-transformed fibroblast cell lines (see, e.g. [28]). 


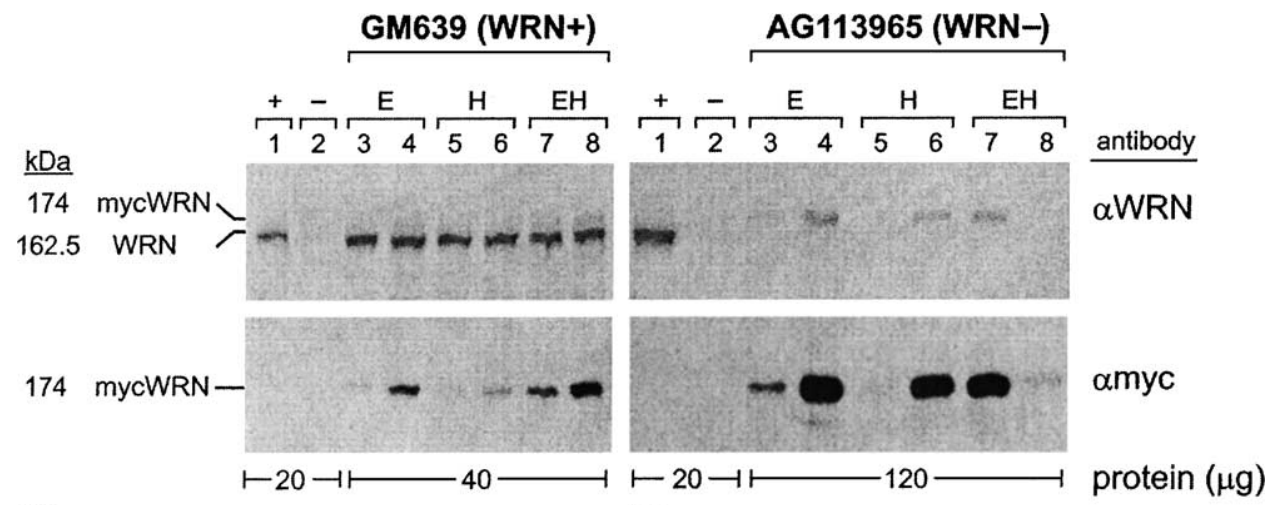

(A)

(B)

Fig. 4. Western blot analysis of WRN transgene expression in stable transfectants. WRN transgene expression in the pairs of independently derived sublines in the same order shown in Fig. 3 with WRN (upper panels) or myc (lower panels) antibody. Uninduced transgene expression levels ranged from $\sim 2.5$ to $25 \%$ of wildtype levels after correcting for signal intensity and the different amounts of protein loaded (40 $\mu \mathrm{g}$ left panels or $120 \mu \mathrm{g}$ right panels). Extract controls consisted of $20 \mu \mathrm{g}$ of total protein from control (WRN+) and WS cell lines (left two lanes in all panels).

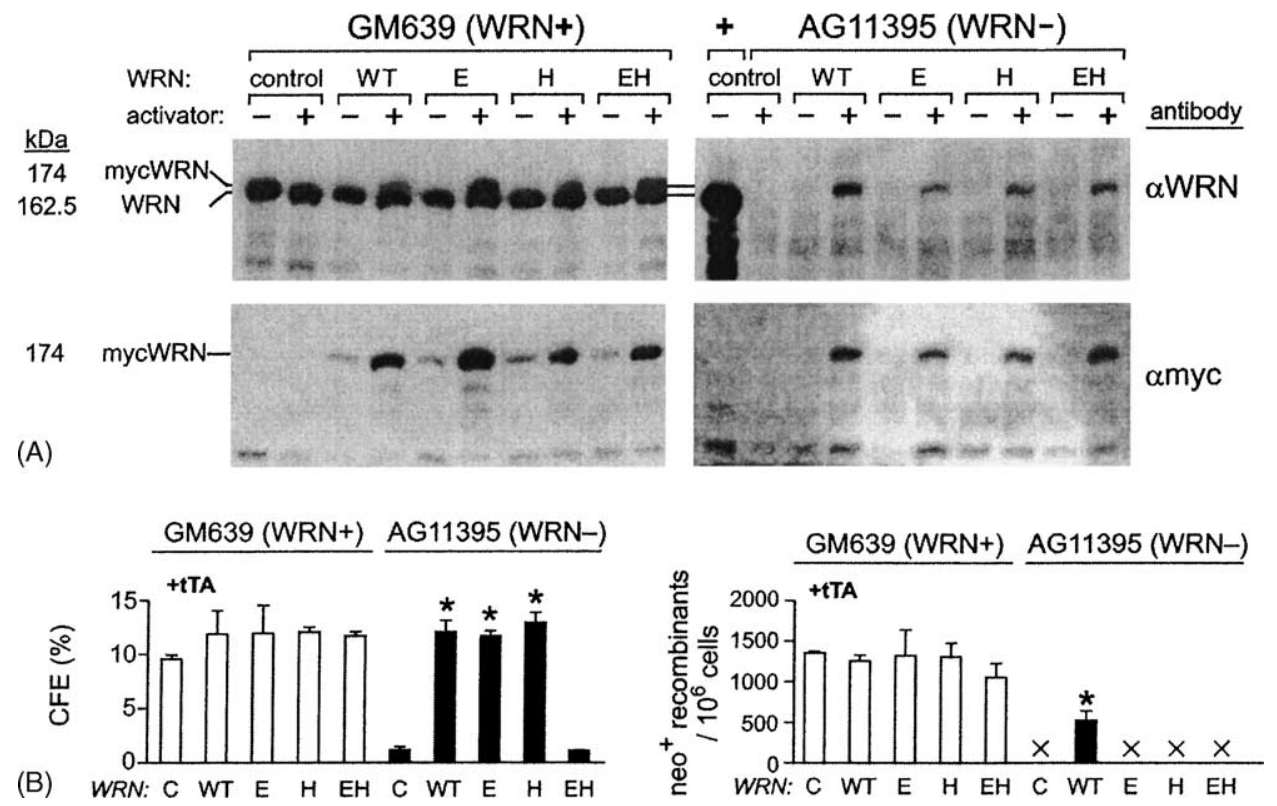

Fig. 5. Cell survival and recombination are not affected by WRN expression level. (A) Western blot analysis of WRN transgene expression in control and WS cells transiently transfected with WRN coding plasmids (Fig. 1A) and a tTA activator plasmid (activator; [14]). WRN expression, detected with WRN (upper panels) or myc epitope tag (lower panels) antibodies, increased in all induced clones. (B) Increased expression of WRN did not improve survival or recombination of WS cells after DNA damage (compare Figs. 2 and 5B). Error bars are standard deviations for a minimum of two replicate experiments. key: $(*)$ statistically significant recovery (see text for $P$ values).

\section{Discussion}

Our results indicate that human WRN protein has recombination and survival functions that differ in their requirement for WRN exonuclease and helicase activities. Recombination is the more stringent of the functions, and requires both activities; either activity, in contrast, supported cell survival after DNA damage. WRN is not unique in having recombination and survival functions that can be separated by mutations. Similar examples are known, among which the most potentially instructive involve members of the RecA/RAD51 strand transferase family (see, e.g. [29]; reviewed in [30,31]).
One important function for WRN in somatic cells appears to be in the resolution of intra-chromosomal recombination products. Single missense-mutant forms of WRN display the same reduced rate of generation of recombinant daughter cells as previously observed in cells lacking WRN protein (Figs. 2, 3 and 5; [12,13]). The likely substrate for WRN in these resolution events are D-loops or Holliday junction-containing intermediates that are generated during gene conversion or synthesis-dependent strand annealing [13,32,33].

Biochemical analyses of WRN action on defined oligonucleotide substrates indicate that the biochemical activities of WRN can compete to unwind or degrade different templates, 
and that the outcome is determined by a combination of substrate conformation, the balance of WRN catalytic activities and the presence of additional proteins that have been shown to interact with WRN (reviewed in $[9,10]$ ). For example, the requirement for both WRN catalytic activities in recombination may reflect the ability of WRN and RPA to efficiently unwind strand invasion intermediates such as D-loops [34]. It should be possible to determine whether the WRN catalytic activities are required in the same protein complex to promote the resolution of recombination intermediates in vivo, and whether the over-expression of other RecQ helicase proteins or of alternative $3^{\prime}$ to $5^{\prime}$ exonuclease activities can promote recombination resolution in the absence of, respectively, the WRN helicase or exonuclease activities.

The ability of WRN lacking exonuclease or helicase activity to support cell survival after DNA damage in the absence of recombination was unexpected. One mechanistic inference from these results is that the missense-mutant proteins are likely to be acting on common substrates, as the expression of each single missense-mutant WRN protein gave comparable cell survival frequencies (Figs. 2, 3 and 5). These common substrates are likely to include recombination intermediates or products $[12,13]$.

The biochemical properties of the WRN helicase and exonuclease suggest several models to explain how each activity in isolation could promote cell survival in the absence of recombination (Fig. 6). Isolated exonuclease or helicase activity could, respectively, degrade or unwind recombination intermediates or products that could not otherwise be correctly resolved. This would topologically disentangle the molecules and generate DNA ends that could reinvade or be captured for DNA end joining (Fig. 6A). Alternatively, the WRN exonuclease or helicase activities could process or stabilize recombination or replication intermediates to promote replication restart, lesion bypass or DNA cleavage (Fig. 6B and C) $[33,35]$. Each pathway is plausible in light of known biochemical properties and actions of the WRN catalytic activities in vitro (reviewed in $[9,10]$ ). Additional biochemical requirements in each of the above survival pathways could be fulfilled by RecQ helicases, topoisomerases, or replication and recombination proteins that have been shown to interact with WRN $[7,10]$. It is important to emphasize that recombination-independent survival pathways are likely to be error-prone: thus cell survival in the absence of recombination would come at the cost of genome instability.

Our results indicate that both WRN catalytic activities must be lost to give rise to the WS cellular phenotype. The loss of both activities is thus likely required to promote WS pathogenesis. A requirement for the coordinate loss of both of the WRN catalytic activities to fully reveal the WS cellular phenotype provides a facile explanation for the spectrum of WRN mutations found in WS patients. This spectrum consists solely of mutations that lead to WRN loss [11]. A second, less obvious, conclusion is that WRN is unlikely to harbor additional activities that strongly influence cell survival or recombination. This is indicated by the virtually identical recombination and survival phenotypes of WS cells that lack detectable WRN protein, and of the same cells expressing catalytically inactive (EH-mutant) WRN protein (Figs. 2, 3, 5and Fig. 1 in Supplementary Material). Two alternative explanations that could explain this apparent lack of 'scaffolding' activity are low or no expression of the

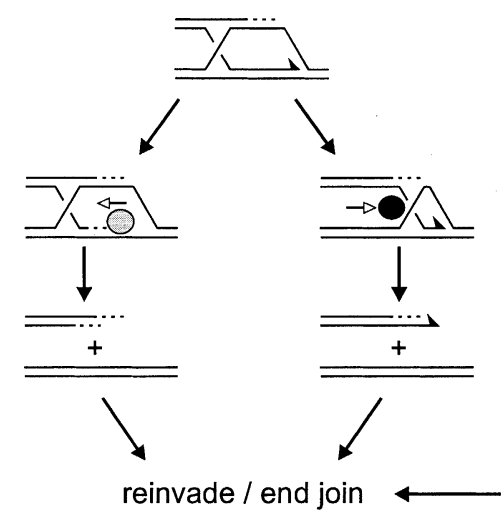

(A)

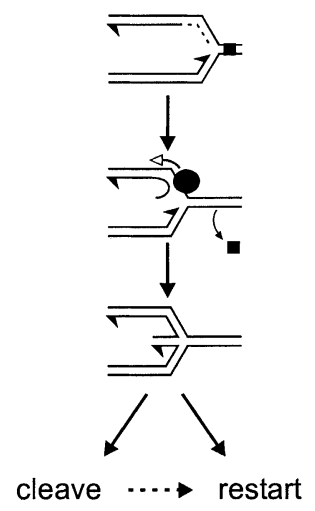

(B)

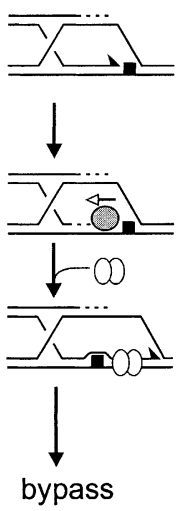

(C)

\begin{tabular}{|ll|}
\hline WRN helicase & O bypass polymerase \\
WRN exonuclease & DNA damage \\
\hline
\end{tabular}

Fig. 6. Potential survival pathways utilizing WRN exonuclease or helicase activity in the absence of recombination. (A) Degradation or unwinding of

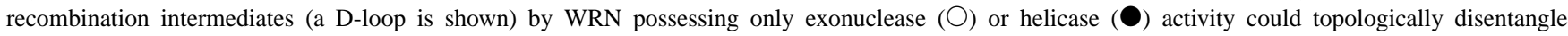
molecules for reinvasion or end-joining. (B) Degradation or unwinding of replication forks stalled by DNA damage ( $\square$ ) could remove bound proteins, stabilize the fork for restart after repair, or promote regression and cleavage to generate free DNA ends. Lagging strand unwinding is shown as an example. (C) Disassembly or unwinding of a replication complex stalled at template DNA damage during recombination. WRN exonuclease or helicase activity could promote assembly of a bypass complex (open ovals) containing one or more specialized DNA polymerases. All three pathways depicted here are likely to be error-prone (see text). 
EH-mutant protein or mislocalization of the mutant protein. Both are unlikely in light of our Western data and the ability of each of the single missense-mutant proteins to efficiently promote cell survival after DNA damage.

The most intriguing implication of our work is that WRN missense mutations or polymorphisms that affect catalytic activity have the potential to promote human disease risk by interfering with recombination. This mechanism may be important in the general population as WS-associated WRN mutations are common (to $<1 / 100$ in, e.g. Japan; [2], and over $375 W R N$ polymorphisms and potential missense mutations of uncertain functional importance have already been identified ([11]; additional unpublished results).

Clinical expression of selective loss of the WRN recombination function would most likely be a genetic instability or cancer predisposition syndrome. One place to look for such selective WRN functional deficits in association with WRN missense mutations or polymorphisms is in otherwise normal individuals who have multiple primary neoplasms of the types observed in WS patients, e.g. osteosarcoma [36,37]. If identified, additional $W R N$-linked recombination deficiency syndromes would join WS and a growing number of human disease predispositions that result from defects in recombination pathways that act to insure genomic stability and suppress the risk of cancer [38,39].

\section{Acknowledgements}

We thank members of the Monnat Lab and University of Washington Program on 'Genetic Instability in Werner Syndrome' for help with experiments and insightful comments on the manuscript. Graphics were generated by A.F.M. Hackmann. This work was supported by the NCI (CA077852); by Roman Undergraduate and Mary Gates Undergraduate Research Scholars Awards to C.S.; by Fellowship Awards from the Ligue Nationale Contre le Cancer and the Philippe Foundation to Y.S.; by an NIH R29 Award to M.J. Emond (CA77607), and by the Nippon Boehringer Ingelheim Virtual Research Institute on Aging to R.J. Mommat Jr.

\section{References}

[1] C.J. Epstein, G.M. Martin, A.L. Schultz, A.G. Motulsky, Werner's syndrome: a review of its symptomatology, natural history, pathologic features, Medicine 45 (1966) 177-221.

[2] G.D. Schellenberg, T. Miki, C.-E. Yu, J. Nakura, Werner syndrome, in: C.R. Scriver, A.L. Beaudet, W.S. Sly, D. Valle (Eds.), The Metabolic and Molecular Basis of Inherited Disease, McGraw-Hill, New York, 2001, pp. 785-797.

[3] M. Goto, R.W. Miller, Y. Ishikawa, H. Sugano, Excess of rare cancers in Werner syndrome (adult progeria), Cancer Epidemiol. Biomarkers Prev. 5 (1996) 239-246.

[4] M. Goto, Hierarchical deterioration of body systems in Werner's syndrome: implications for normal ageing, Mech. Ageing Dev. 98 (1997) 239-254.
[5] C.-E. Yu, J. Oshima, Y.-H. Fu, E.M. Wijsman, F. Hisama, S. Ouais, J. Nakura, T. Miki, G.M. Martin, J. Mulligan, G.D. Schellenberg, Positional cloning of the Werner's syndrome gene, Science 272 (1996) 258-262.

[6] H. Nakayama, RecQ family helicases: roles as tumor suppressor proteins, Oncogene 21 (2002) 9008-9021.

[7] I.D. Hickson, RecQ helicases: caretakers of the genome, Nat. Rev. Cancer 3 (2003) 169-178.

[8] A.J. van Brabant, R. Stan, N.A. Ellis, DNA helicases, genomic instability, and human genetic disease, Annu. Rev. Genomics Hum. Genet. 1 (2000) 409-459.

[9] J.-C. Shen, L.A. Loeb, The Werner syndrome gene: the molecular basis of RecQ helicase-deficiency diseases, Trends Genet. 16 (2000) 213-220.

[10] P.L. Opresko, W.-H. Cheng, C. von Kobbe, J.A. Harrigan, V.A. Bohr, Werner syndrome and the function of the Werner protein what they can teach us about the molecular aging process, Carcinogenesis 24 (2003) 791-802.

[11] P.W. Wagner, R.J. Monnat Jr., Werner syndrome mutational database, Release 2. http://www.pathology.washington.edu/research/ werner/ws_wrn.html.2003.

[12] P.R. Prince, M.J. Emond, R.J. Monnat Jr., Loss of Werner syndrome protein function promotes aberrant mitotic recombination, Genes Dev. 15 (2001) 933-938.

[13] Y. Saintigny, K. Makienko, C. Swanson, M.J. Emond, R.J. Monnat Jr., Homologous recombination resolution defect in Werner syndrome, Mol. Cell. Biol. 2 (2002) 6971-6978.

[14] M. Gossen, H. Bujard, Tight control of gene expression in mammalian cells by tetracycline-responsive promoters, Proc. Natl. Acad. Sci. USA 89 (1992) 5547-5551.

[15] G.I. Evan, G.K. Lewis, G. Ramsay, J.M. Bishop, Isolation of monoclonal antibodies specific for the human c-myc proto-oncogene product, Mol. Cell. Biol. 5 (1985) 3610-3616.

[16] M.D. Gray, J.-C. Shen, A.S. Kamath-Loeb, A. Blank, B.L. Sopher, G.M. Martin, J. Oshima, L.A. Loeb, The Werner syndrome protein is a DNA helicase, Nat. Genet. 17 (1997) 100-103.

[17] S. Huang, B. Li, M.D. Gray, J. Oshima, I.S. Mian, J. Campisi, The premature aging syndrome protein, WRN, is a $3^{\prime}$ to $5^{\prime}$ exonuclease, Nat. Genet. 20 (1998) 114-116.

[18] M.J. Moser, A.S. Kamath-Loeb, J.E. Jacob, S.E. Bennett, J. Oshima, R.J. Monnat Jr., WRN helicase expression in Werner syndrome cell lines, Nucleic Acids Res. 28 (2000) 648-654.

[19] F.S. Wyllie, C.J. Jones, J.W. Skinner, M.F. Haughton, C. Wallis, D. Wynford-Thomas, R.G.A. Faragher, D. Kipling, Telomerase prevents the accelerated cell ageing of Werner syndrome fibroblasts, Nat. Genet. 24 (2000) 16-17.

[20] F. Hisama, Y.-H. Chen, M.S. Meyn, J. Oshima, S.M. Weissman, WRN or telomerase constructs reverse 4-nitroquinoline 1-oxide sensitivity in transformed Werner syndrome fibroblasts, Cancer Res. 60 (2000) 2372-2376.

[21] M. Poot, J.S. Yom, S.H. Whang, J.T. Kato, K.A. Gollahon, P.S. Rabinovitch, Werner syndrome cells are sensitive to DNA cross-linking drugs, FASEB J. 15 (2001) 1224-1226.

[22] K.J. Yarema, S.J. Lippard, J.M. Essigmann, Mutagenic and genotoxic effects of DNA adducts formed by the anticancer drug cis-diammine dichloroplatinum (II), Nucleic Acids Res. 23 (1995) 4066-4072.

[23] M.S. Meyn, High spontaneous intrachromosomal recombination rates in ataxia-telangiectasia, Science 260 (1993) 1327-1330.

[24] C.E. Ogburn, J. Oshima, M. Poot, R. Chen, K.E. Hunt, K.A. Gollahon, P.S. Rabinovitch, G.M. Martin, An apoptosis-inducing genotoxin differentiates heterozygotic carriers for Werner helicase mutations from wild-type and homozygous mutants, Hum. Genet. 101 (1997) 121-125.

[25] M. Poot, K.A. Gollahon, P.S. Rabinovitch, Werner syndrome lymphoblastoid cells are sensitive to camptothecin-induced apoptosis in S-phase, Hum. Genet. 104 (1999) 10-14. 
[26] M.J. Moser, W.L. Bigbee, S.G. Grant, M.J. Emond, R.G. Langlois, R.H. Jensen, J. Oshima, R.J. Monnat Jr., Genetic instability and hematologic disease risk in Werner syndrome patients and heterozygotes, Cancer Res. 60 (2000) 2492-2496.

[27] T. Kawabe, N. Tsuyama, S. Kitao, K. Nishikawa, A. Shimamoto, M. Shiratori, T. Matsumoto, K. Anno, T. Sato, Y. Mitsui, M. Seki, T. Enomoto, M. Goto, N.A. Ellis, T. Ide, Y. Furuichi, M. Sugimoto, Differential regulation of the human RecQ family helicases in cell transformation and cell cycle, Oncogene 19 (2000) 4764-4772.

[28] T.H.-T. Chang, F.A. Ray, D.A. Thompson, R. Schlegel, Misregulation of mitotic checkpoints and regulatory proteins following acute expression of SV40 large T antigen in diploid human cells, Oncogene 14 (1997) 2383-2393.

[29] C. Morrison, A. Shinohara, E. Sonoda, Y. Yamaguchi-Iwai, M. Takata, R.R. Weichselbaum, S. Takeda, The essential functions of human Rad51 are independent of ATP hydrolysis, Mol. Cell. Biol. 19 (1999) 6891-6897.

[30] L.H. Thompson, D. Schild, Homologous recombinational repair of DNA ensures mammalian chromosome stability, Mutat. Res. 477 (2001) 131-153.

[31] L.S. Symington, Role of RAD52 epistasis group gene in homologous recombination and double-strand break repair, Microbiol. Mol. Biol. Rev. 66 (2002) 630-670.

[32] F. Pâques, J.E. Haber, Multiple pathways of recombination induced by double-strand breaks in Saccharomyces cerevisiae, Microbiol. Mol. Biol. Rev. 63 (1999) 349-404.
[33] W.-D. Heyer, K.T. Ehmsen, J.A. Solinger, Holliday junctions in the eukaryotic nucleus: resolution in sight? Trends Biochem. Sci. 28 (2003) 548-557.

[34] P.L. Opresko, J.-P. Laine, R.M. Brosh Jr., M.M. Seidman, V.A. Bohr, Coordinate action of the helicase and $3^{\prime}$ to $5^{\prime}$ exonuclease of Werner syndrome protein, J. Biol. Chem. 276 (2001) 44677 44687.

[35] P. McGlynn, R.G. Lloyd, Recombinational repair and restart of damaged replication forks, Nat. Rev. Mol. Cell. Biol. 3 (2002) 859 870.

[36] M. Verneris, I.R. McDougall, D. Becton, M.P. Link, Thyroid carcinoma after successful treatment of osteosarcoma: report of three patients, J. Pediatr. Hematol. Oncol. 23 (2001) 312-315.

[37] E.I. Hauben, J. Arends, J.P. Vandenboucke, C.J. van Asperen, E. Van Marck, P.C.W. Hogendoorn, Multiple primary malignancies in osteosarcoma patients. Incidence and predictive value of osteosarcoma subtype for cancer syndromes related with osteosarcoma., Eur. J. Hum. Genet. 11 (2003) 611-618.

[38] A.J. Pierce, J.M. Stark, F.D. Araujo, M.E. Moynahan, M. Berwick, M. Jasin, Double-strand breaks and tumorigenesis, Trends Cell Biol. 11 (2001) 52-59.

[39] L.H. Thompson, D. Schild, Recombinational DNA repair and human disease, Mutat. Res. 509 (2002) 49-78.

[40] R.A.W. Rupp, L. Snider, H. Weintraub, Хеnopus embryos regulate the nuclear localization of XMyoD, Genes Dev. 8 (1994) 13111323. 